\section{Ageing Effect on Phage Particles leading to an Increase in Burst Size}

IN constant conditions, the burst size of a particular bacteriophage is a characteristic of that bacteriophage. We have found aged $P 4 / 2$ phage particles yield larger burst sizes than young phage particles.

The bacteriophage $P 4 / 2$ was isolated from Salmonella potsdam (NCTC 3206) and propagated on another strain of $S$. potsdam, $S P 2$, which seems to be non-lysogenic ${ }^{1}$. Phage lysates were stored in a refrigerator, and at periodic intervals one-step growth experiments were performed in which the multiplicities of infection were between 0.01 and $0 \cdot 1$. A steady increase in the average burst size was found with an initial low value of $\mathbf{3 4}$ from cells infected with fresh phage particles to a high value of 786 for cells infected with phage particles which were 1 month old. The burst sizes from cells infected with phage stored for times longer than 1 month were approximately the same as those obtained with cells infected with month old phage. The rate of adsorption, determined by measure of both unadsorbed phage and of infective centres, was found to be independent of the age of the infecting phage particles. Neither the rate of adsorption nor the burst size itself was affected by variations in the multiplicity of infection. The burst sizes produced by phage lysates kept at both higher and lower temperatures were like those yielded by phage kept for the same time in a refrigerator even though there was a drop in the plaque forming titre of lysates kept at higher temperatures.

In a series of single burst experiments, the proportion of infected cells which gave rise to small numbers of phage progeny was high when young phage particles were used for infection but low when old phage particles were used. The proportion of cells infected with aged phage particles, which yielded large bursts, was also significantly highor. These results support those obtained in the one-step growth experiments but give the added information that the differences in the average burst size are directly related to the size of the burst from individual cells rather than to differences in the proportion of cells which release phage progeny.

A series of premature lysis experiments was performed with lysates which gave bursts of different sizes. The length of the eclipse phase was found to be shortened as the burst size increased although the latent period itself remained constant at $40 \mathrm{~min}$. The shortest eclipse phase was $20 \mathrm{~min}$ for a burst of 700 , the longest was $35 \mathrm{~min}$ for a burst of 34. Thus the length of the eclipse phase was inversely proportional to the size of the burst.

If there is the same relationship between phage DNA and the appearance of mature, intracellular phage with this phage and its host as there is with $T 2$ infected cells of $E$. coli, a delay in the appearance of intracellular phage would mean a delay in the synthesis of $\mathrm{DNA}^{2}$. Attempts to test this directly have so far failed because, at the low multiplicities of infection necessary to prevent lysogeniza. tion, the net DNA synthesis was not representative of phage infected cells. We cannot therefore determine whether the delay which results in initial, small average burst sizes attributed to one or more phage regulated functions is associated with a delay in the onset of phage DNA synthesis or in the maturation process with a consequently larger DNA vegetative pool.

This investigation was carried out under contract from the US Office of Naval Research.

LeONARd R. Bullas

ANTHONY J. ZUCCARELLI

ROBERT L. NUTTER

Department of Microbiology, Medical School,

Loma Linda University,

Loma Linda, California.

Received September 29; revised November 17, 1967.

${ }^{1}$ Atkinson, N., and Bullas, L. R., Austral. J. Exp. Biol. Med. Sci., 34, 461 (1956).

'Nutter, R. L., and Sinsheimer, R. I., Virology, 7, 276 (1959).

\section{Distribution of the Soluble Dehydrogenases and Diaphorases of Brucei Subgroup of Trypanosomes in Starch-gel Electropherograms}

ANTIGENIC variation in the brucei subgroup of trypano. somes considerably limits the development of a suitable anti-trypanosomal vaccine, and this limitation is likely to continue until we have more knowledge of the nature of antigenic variation. Until then, however, more information about the nature of trypanosome antigens would be useful.

Some of the variable antigens are soluble proteins and the variation seems to be associated with changes in their immunological specificity ${ }^{1-3}$. We have separated soluble trypanosome proteins into at least twenty-nine components by starch.gel electrophoresis ${ }^{4}$, and in an attempt to characterize these components we tested for glucose-6-phosphate dehydrogenase which was known to be present ${ }^{5}$. This was found in the region occupied by the major $4 S$ variable antigens of Williamson and Brown ${ }^{2}$ (bands 1-7 in Fig. 1). This suggested that the antigens might be dehydrogenases, and so we made a survey of some of the dehydrogenases and diaphorases of carbohydrate metabolism. We present here the results of the distribution of these enzymes in starch-gel electropherograms and discuss their possible role in metabolism.

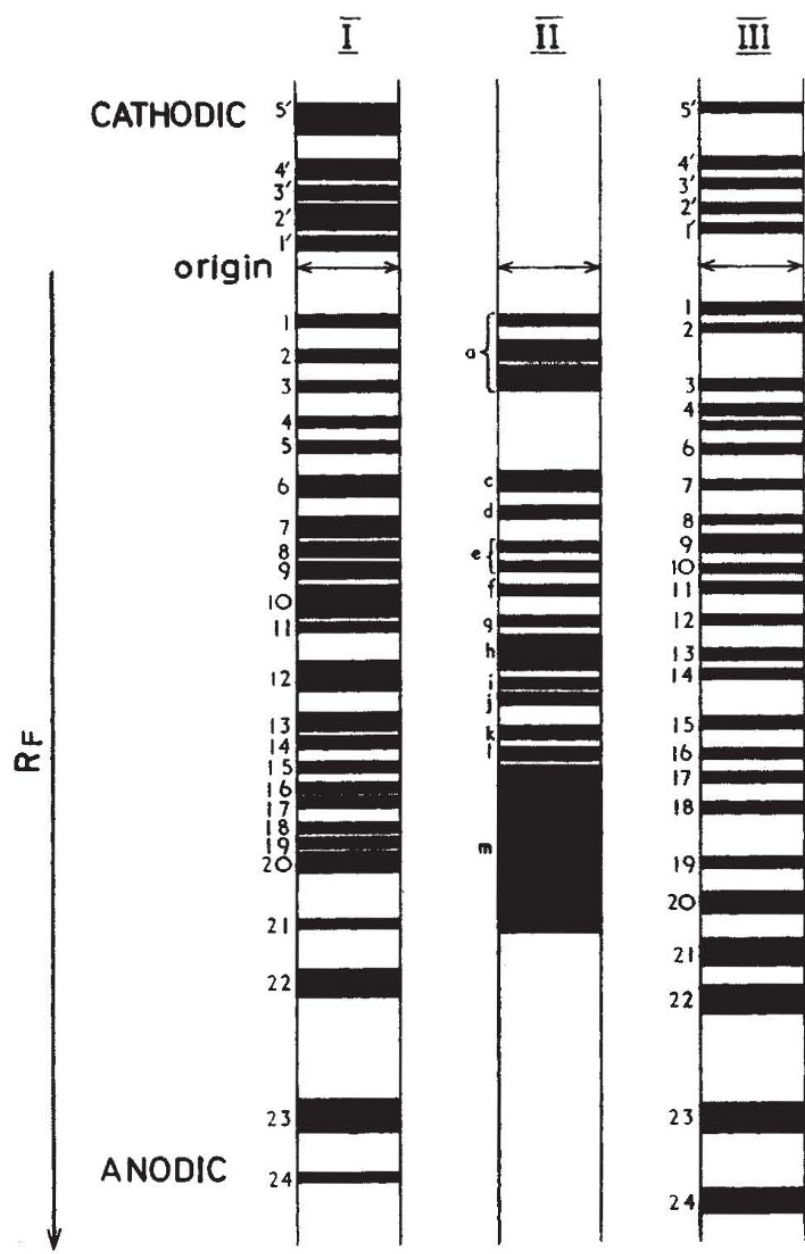

Fig. 1. Starch-gel electropherogram of the rat serum and soluble trypanosome proteins. I, EATRO-691; II, normal rat serum; III,
$T$. rhodesiense, SS 518. The figure is a combined scale drawing from different preparations run at different times. The rat serum notation is that of Beaton et al. ${ }^{13}$. 0.05 molar glycine/sodium hydroxide buffer, $p H$ 8.9, gel; 0.05 molar borate buffer, $p$ H 8.58, electrode chambers; $8 \mathrm{~V} / \mathrm{cm}, 2 \cdot 5 \mathrm{~h}$. Proteins stained in 1 per cent amido black $10 B$ in methanol/water/acetic acid $(50 / 50 / 10, v / v)$. 